\title{
Character Sums with Division Polynomials
}

\author{
IGOR E SHPARLINSKI AND KATHERINE E. STANGE
}

\begin{abstract}
We obtain nontrivial estimates of quadratic character sums of division polynomials $\Psi_{n}(P), n=1,2, \ldots$, evaluated at a given point $P$ on an elliptic curve over a finite field of $q$ elements. Our bounds are nontrivial if the order of $P$ is at least $q^{1 / 2+\varepsilon}$ for some fixed $\varepsilon>0$. This work is motivated by an open question about statistical indistinguishability of some cryptographically relevant sequences which has recently been brought up by K. Lauter and the second author.
\end{abstract}

\section{Division Polynomials and Character Sums}

Let $E$ be an elliptic curve over a finite field $\mathbb{F}_{q}$ of characteristic $p>3$. Denote by $E\left(\mathbb{F}_{q}\right)$ the group of points of $E$ defined over $\mathbb{F}_{q}$. We refer to [9] for background on elliptic curves.

Let $\Psi_{n}$ be the $n$-th division polynomial for positive integers $n$. For a given point $P \in E\left(\mathbb{F}_{q}\right)$, the sequence $\Psi_{n}(P)$ is often called an elliptic divisibility sequence. It satisfies the following recurrence relation [9, Exercise 3.34]

$$
\begin{aligned}
& \Psi_{h+i}(P) \Psi_{h-i}(P) \Psi_{j}(P)^{2} \\
& +\Psi_{i+j}(P) \Psi_{i-j}(P) \Psi_{h}(P)^{2} \\
& \quad+\Psi_{j+h}(P) \Psi_{j-h}(P) \Psi_{i}(P)^{2}=0
\end{aligned}
$$

By definition, $\Psi_{n}(P)=0$ if and only if $[n] P=0$. Further, the sequence $\Psi_{n}(P)$ is necessarily periodic with some period $T$ and $T$ is always a multiple of the order of $P$ (see Lemma 1 1below). For background on elliptic divisibility sequences, see [2, 11, 12].

Note that elliptic divisibility sequences can be viewed as a generalisation of Lucas sequences. We recall that Lucas sequences (of the first kind) are sequences satisfying a recurrence of the form

$$
L_{n}=a L_{n-1}+b L_{n-2}, \quad L_{0}=0, \quad L_{1}=1,
$$

Date: August 11, 2021.

1991 Mathematics Subject Classification. 11L40, 14H52.

Key words and phrases. division polynomial, character sum. 
in given coefficients $a$ and $b$. Lucas sequences, including Fibonacci numbers, satisfy (11) after an appropriate scaling (multiplication of the $n$-th term by $\lambda^{n^{2}-1}$ for some $\lambda$ ); see [9, Exercise 3.34] and [12, Section VI].

In this paper, for a fixed point $P \in E\left(\mathbb{F}_{q}\right)$, and an integer $N \leq T$, we obtain nontrivial estimates of sums of the form

$$
S_{P}(N)=\sum_{n=1}^{N} \chi\left(\Psi_{n}(P)\right),
$$

where $\chi$ is the quadratic character of $\mathbb{F}_{q}$ (as usual, we set $\chi(0)=0$ ). Character sums with linear recurrence sequences have been studied in [8]. See also [2, Chapter 5] for a survey of estimates of exponential and character sums with various recurrence sequences. However, to our knowledge, for elliptic divisibility sequences no results have been obtained prior to this work.

\section{Motivation}

This question also has a cryptographic connection. In [5] the following elliptic divisibility sequence residue problem has been considered: given two points $P, Q \in E\left(\mathbb{F}_{q}\right)$ such that $Q \in\langle P\rangle, Q \neq \mathcal{O}$ and $\operatorname{ord}(P) \geq 4$, calculate $\chi\left(\Psi_{k}(P)\right)$ for the smallest positive $k$ such that $Q=[k] P$. To find $k$ given the points $P$ and $Q$ is the well-known elliptic curve discrete logarithm problem and its assumed difficulty is the basis of elliptic curve cryptography. To solve the residue problem it certainly suffices to solve the discrete logarithm problem. However, it may be possible to solve the residue problem without first calculating $k$. It is shown in [5, Theorem 1.1] that solving either of these problems in subexponential time leads to a solution of the other in subexponential time. For perspective, the calculation of $\chi\left(\Psi_{k+1}(P) / \Psi_{k}(P)\right)$ takes only polynomial time from $P$ and $Q$, and does not reveal $k$, see [5, Section 8]. This has raised the general question of what can be said about the residuosity of $\Psi_{n}(P)$. More specifically, it has been shown in [5] that the difficulty of a certain distinguishability problem of cryptographic interest depends on the bias between the quadratic residues and nonresidues amongst consecutive terms of the sequence $\Psi_{n}(P)$, $n=1, \ldots, N$, which is in turn is equivalent to estimating the sums $S_{P}(N)$.

\section{Prerequisites CONCERning Division polynomials}

We recall some classical results, the first of which describes the ratio $\Psi_{n+r}(P) / \Psi_{n}(P)$.

By [10, Theorem 8] (see also [12, Theorem 8.1]), we have: 
Lemma 1. Let $P \in E\left(\mathbb{F}_{q}\right)$ be of order $r \geq 3$. Then for all positive $s, k \in \mathbb{Z}$,

$$
\Psi_{s r+k}(P)=a^{k s} b^{s^{2}} \Psi_{k}(P),
$$

where $a$ and $b$ are given by

$$
a=\frac{\Psi_{r-2}(P)}{\Psi_{r-1}(P) \Psi_{2}(P)}, \quad b=\frac{\Psi_{r-1}(P)^{2} \Psi_{2}(P)}{\Psi_{r-2}(P)} .
$$

Furthermore, by [10, Lemma 6], we also have:

Lemma 2. Let $n$ and $m$ be positive integers. Then

$$
\Psi_{n m}(P)=\Psi_{n}([m] P) \Psi_{m}(P)^{n^{2}} .
$$

We remark that in general, for $P \in E\left(\mathbb{F}_{q}\right)$ of order $r \geq 3$, the period $T$ of the the sequence $\Psi_{n}(P)$ may be as large as $r(q-1)$, see [10, Corollary 9]. In turn, $r$ can be of order $q$ as well, for example, if $P$ is a generator of the cyclic group of points.

However, the following result, that is immediate from Lemma 1 , shows that the sequence $\chi\left(\Psi_{n}(P)\right)$ is of smaller period.

Lemma 3. Let $P \in E\left(\mathbb{F}_{q}\right)$ be of order $r \geq 3$. Then the sequence $\chi\left(\Psi_{n}(P)\right)$ is periodic with period which is a divisor of $R=2 r$.

Thus, we see from Lemma 3 that bounds of character sums $S_{P}(N)$ are of interest only for the values of $N \leq R=2 r$.

\section{Prerequisites concerning Character sums}

It is well-known that for an elliptic curve $E$ over $\mathbb{F}_{q}$ we have

$$
E\left(\mathbb{F}_{q}\right) \sim \mathbb{Z} / M \mathbb{Z} \times \mathbb{Z} / L \mathbb{Z}
$$

for unique integers $M$ and $L$ satisfying $L \mid M$. The point $P$ and $Q$ are called echelonized generators if $P$ has order $M, Q$ has order $L$ and any point of $E\left(\mathbb{F}_{q}\right)$ can be written in the form $m P+\ell Q$ with $1 \leq m \leq M$ and $1 \leq \ell \leq L$.

Let $\Omega=\operatorname{Hom}\left(E(k), \mathbb{C}^{*}\right)$ be the group of characters on $E(k)$; this is given explicitly by

$$
\Omega=\left\{\mathbf{e}_{M}(a m) \mathbf{e}_{L}(b \ell): 0 \leq a<M, 0 \leq b<L\right\},
$$

where for a positive integer $K$, we define

$$
\mathbf{e}_{K}(z)=\exp (2 \pi i z / K) \text {. }
$$

The following multiplicative analogue of a result of [4] is essentially [1, Proposition 1], which in turns comes from [6] (note that in [1] it is formulated only for prime fields but the proof extends to arbitrary fields without any difficulties). 
Lemma 4. Let $\eta$ be a non-principal multiplicative character on $\mathbb{F}_{q}^{*}$. Let $\mathbb{K}=\mathbb{F}_{q}(E)$ be the function field of an elliptic curve $E$ over $\mathbb{F}_{q}$, and $f \in \mathbb{K}$ be of degree $d$ and such that $f \neq g^{m}$ for any function $g$ in the algebraic closure $\overline{\mathbb{K}}$ of $\mathbb{K}$ and $m \mid q-1$. Let $\omega \in \Omega$. Then

$$
\left|\sum_{P \in E\left(\mathbb{F}_{q}\right)}^{*} \omega(P) \eta(f(P))\right| \leq 2 d \sqrt{q}
$$

where $\sum^{*}$ indicates that the sum is over $P \in E\left(\mathbb{F}_{q}\right)$ such that $f(P) \neq$ $\infty$.

Lemma 5. Under the assumptions of Lemma 4, let $H \subseteq E\left(\mathbb{F}_{q}\right)$ be a subgroup. Then

$$
\left|\sum_{P \in H}^{*} \omega(P) \eta(f(P))\right| \leq 2 d \sqrt{q}
$$

where $\sum^{*}$ indicates that the sum is over $P \in H$ such that $f(P) \neq \infty$.

Proof. Let $\Omega_{H} \subseteq \Omega$ be the subset of characters $\vartheta$ such that $H \subseteq \operatorname{ker}(\vartheta)$. Then, $\Omega_{H}$ is dual to $E\left(\mathbb{F}_{q}\right) / H$, so by the orthogonality property of characters of abelian groups, we have

$$
\frac{1}{\left|\Omega_{H}\right|} \sum_{\vartheta \in \Omega_{H}} \vartheta(P)= \begin{cases}1 & P \in H \\ 0 & P \notin H .\end{cases}
$$

Therefore

$$
\begin{aligned}
\sum_{P \in H}^{*} \omega(P) \eta(f(P)) & =\frac{1}{\left|\Omega_{H}\right|} \sum_{P \in E\left(\mathbb{F}_{q}\right)}^{*} \sum_{\vartheta \in \Omega_{H}} \vartheta(P) \omega(P) \eta(f(P)) \\
& =\frac{1}{\left|\Omega_{H}\right|} \sum_{\vartheta \in \Omega_{H}}\left(\sum_{P \in E\left(\mathbb{F}_{q}\right)}^{*} \vartheta \cdot \omega(P) \eta(f(P))\right)
\end{aligned}
$$

Applying Lemma 4, we obtain the desired result.

\section{MAin RESUlTS}

Here we estimate the incomplete sum $S_{P}(N)$. Following the standard approach we start with estimates of complete sums twisted with an additive character.

As before, let $R=2 r$ where $r$ is the order of $P$. Then for an integer $a$ we define the sums

$$
T_{P}(a)=\sum_{n=1}^{R} \chi\left(\Psi_{n}(P)\right) \mathbf{e}_{R}(a n) .
$$


which can be of independent interest.

Theorem 6. For any integer a, we have

$$
T_{P}(a)=O\left(R^{5 / 6} q^{1 / 12}(\log q)^{1 / 3}\right) .
$$

Proof. Let $a \in \mathbb{Z}$. Fix an integer $L \geq 3$ and let $\mathcal{L}$ denote the set of odd primes $\ell$ such that $\ell<L$ and $\ell \nmid R$. Since $R$ has at most $O(\log R)=O(\log q)$ prime divisors we see, say, for

$$
L \geq(\log q)^{2}
$$

and sufficiently large $q$ we have

$$
\# \mathcal{L} \geq \frac{L}{2 \log L} .
$$

Let $\ell \in \mathcal{L}$. As $n$ runs through the all residue classes modulo $R$, so does $\ell n$. Since both sequences $\chi\left(\Psi_{n}(P)\right)$ and $\mathbf{e}_{R}($ an $), n=1,2, \ldots$, are periodic with period $R$, we have

$$
T_{P}(a)=\sum_{n=1}^{R} \chi\left(\Psi_{\ell n}(P)\right) \mathbf{e}_{R}(a \ell n) .
$$

We average over all choices of $\ell \in \mathcal{L}$ and set

$$
W=\sum_{\ell \in \mathcal{L}} \sum_{n=1}^{R} \chi\left(\Psi_{\ell n}(P)\right) \mathbf{e}_{R}(a \ell n) .
$$

Then we have

$$
T_{P}(a)=\frac{1}{\# \mathcal{L}} W
$$

To estimate $W$, we change the order of summation, and then apply the Cauchy inequality:

$$
|W|^{2} \leq R \sum_{n=1}^{R}\left|\sum_{\ell \in \mathcal{L}} \chi\left(\Psi_{\ell n}(P)\right) \mathbf{e}_{R}(a \ell n)\right|^{2} .
$$

Now we apply Lemma 2;.

$$
\begin{aligned}
|W|^{2} & \leq R \sum_{n=1}^{R}\left|\sum_{\ell \in \mathcal{L}} \chi\left(\Psi_{\ell n}(P)\right) \mathbf{e}_{R}(a \ell n)\right|^{2} \\
& =R \sum_{n=1}^{R}\left|\sum_{\ell \in \mathcal{L}} \chi\left(\Psi_{\ell}(n P)\right) \chi\left(\Psi_{n}(P)^{\ell^{2}}\right) \mathbf{e}_{R}(a \ell n)\right|^{2} .
\end{aligned}
$$

Since $\chi$ is the quadratic character and $\ell$ is odd, we have

$$
\chi\left(\Psi_{n}(P)^{\ell^{2}}\right)=\chi\left(\Psi_{n}(P)\right) .
$$


Therefore,

$$
\begin{aligned}
|W|^{2} & \leq R \sum_{n=1}^{R}\left|\chi\left(\Psi_{n}(P)\right)\right|^{2}\left|\sum_{\ell \in \mathcal{L}} \chi\left(\Psi_{\ell}(n P)\right) \mathbf{e}_{R}(a \ell n)\right|^{2} \\
& \leq R \sum_{n=1}^{R}\left|\sum_{\ell \in \mathcal{L}} \chi\left(\Psi_{\ell}(n P)\right) \mathbf{e}_{R}(a \ell n)\right|^{2} .
\end{aligned}
$$

Expanding the square and switching the order of summation again, we obtain

$$
\begin{aligned}
|W|^{2} & \leq R \sum_{n=1}^{R} \sum_{\ell_{1}, \ell_{2} \in \mathcal{L}} \chi\left(\Psi_{\ell_{1}}(n P)\right) \mathbf{e}_{R}\left(a \ell_{1} n\right) \chi\left(\Psi_{\ell_{2}}(n P)\right) \mathbf{e}_{R}\left(-a \ell_{2} n\right) \\
& =R \sum_{\ell_{1}, \ell_{2} \in \mathcal{L}} \sum_{n=1}^{R} \chi\left(\Psi_{\ell_{1}}(n P) \Psi_{\ell_{2}}(n P)\right) \mathbf{e}_{R}\left(a\left(\ell_{1}-\ell_{2}\right) n\right) .
\end{aligned}
$$

We now turn to bounding the inner sum.

For $\ell_{1}=\ell_{2}=\ell$, we have the trivial estimate

$$
\sum_{n=1}^{R} \chi\left(\Psi_{\ell}(n P)^{2}\right)<R
$$

For $\ell_{1} \neq \ell_{2}$ we use Lemma 5. The degree of $\Psi_{\ell}(P)$ (considered as a function in the function field of $E)$ is $\left(\ell^{2}-1\right) / 2$, so the degree of $\Psi_{\ell_{1}}(P) \Psi_{\ell_{2}}(P)$ is

$$
\frac{\left(\ell_{1}^{2}+\ell_{2}^{2}-2\right)}{2}<L^{2}-1
$$

It is also easy to see (by examining its zeros) that $\Psi_{\ell_{1}}(P) \Psi_{\ell_{2}}(P)$ is not a square of another function from the same function field. Since by Lemma 3 we have $R \mid 2 r$, we see that

$$
\sum_{n=1}^{R} \chi\left(\Psi_{\ell_{1}}(n P) \Psi_{\ell_{2}}(n P)\right) \mathbf{e}_{R}\left(a\left(\ell_{1}-\ell_{2}\right) n\right)=O\left(L^{2} q^{1 / 2}\right) .
$$

Thus, we obtain

$$
|W|^{2}=O\left(R^{2} \# \mathcal{L}+R L^{2} \sqrt{q}(\# \mathcal{L})^{2}\right) .
$$

Substituting this bound in (4) and using (3), we derive

$$
\begin{aligned}
T_{P}(a) & =O\left(R(\# \mathcal{L})^{-1 / 2}+q^{1 / 4} R^{1 / 2} L\right) \\
& =O\left(R L^{-1 / 2}(\log L)^{1 / 2}+q^{1 / 4} R^{1 / 2} L\right) .
\end{aligned}
$$

We no choose $L=\left\lfloor R^{1 / 3} q^{-1 / 6}(\log q)^{1 / 3}\right\rfloor$, thus (2) is satisfied, provided that $q$ is large enough which implies the desired estimate. 
We remark that Theorem 6] is nontrivial if $R \geq q^{1 / 2+\varepsilon}$ for a fixed $\varepsilon>0$ (we recall that the largest possible value of $R$ is of order $q$ ).

Now using the standard reduction between complete and incomplete sums, see [3, Section 12.2], we obtain

Corollary 7. For any $N \leq R$, we have,

$$
S_{P}(N)=O\left(R^{5 / 6} q^{1 / 12}(\log q)^{4 / 3}\right) .
$$

\section{Comments}

In principle, our approach works for sums of multiplicative characters of arbitrary order $d \mid q-1$. In this case, Lemma 3 needs some obvious adjustments. Furthermore, the set $\mathcal{L}$ in the proof of Theorem 6 has to be chosen to consist of primes $\ell \equiv \pm 1(\bmod d)$, so (5) still holds. For any fixed $d$ the final result is the same, however its strength diminishes as $d$ grows, and for example, for characters of order $q-1$ leads only to a trivial estimate. Although we do not see any immediate cryptographic significance of such a result, obtaining nontrivial estimates of character sums with arbitrary multiplicative characters is a natural and interesting question. A related open question is obtaining nontrivial estimates on similar sums of additive characters of $\mathbb{F}_{q}$. In this case, there is no natural analogue of (5) and thus our approach does not apply at all.

Finally, we mention an algorithmic question which can be of cryptographic relevance. Given a black-box which for every integer $n$ outputs $\chi\left(\Psi_{n}(P)\right)$, the question is to recover the "hidden" point $P$. This admits several modifications depending whether the curve $E$ and the field $\mathbb{F}_{q}$ are known or not. This question is analoguous to the more studied cryptographic problem of recovering a hidden polynomial $f(X) \in \mathbb{F}_{q}[X]$ given a black-box which outputs $\chi(f(n))$; see [7] and references therein.

\section{ACKNOWLEDGEMENT}

The authors would like to thank Kristin Lauter who connected them together and stimulated their joint work on this paper. The authors are also grateful to the Fields Institute for its support and stimulating atmosphere which led to the initiation of this work at the "Fields Cryptography Retrospective Meeting", Toronto, May 2009.

During the preparation of this paper, I. S. was supported in part by ARC Grant DP0881473 and K. S. was supported in part by NSF Fellowship 0802915 and NSERC PDF-373333. 


\section{REFERENCES}

[1] Z. Chen, 'Elliptic curve analogue of Legendre sequences', Monash. Math., 154 (2008), 1-10.

[2] G. Everest, A. J. van der Poorten, I. E. Shparlinski and T. Ward, Recurrence sequences, Amer. Math. Soc., Providence, RI, 2003.

[3] H. Iwaniec and E. Kowalski, Analytic number theory, Amer. Math. Soc., Providence, RI, 2004.

[4] D. R. Kohel and I. E. Shparlinski, 'Exponential sums and group generators for elliptic curves over finite fields', Proc. 4th Algorithmic Number Theory Symposium, Lect. Notes in Comp. Sci., vol. 1838, Springer-Verlag, Berlin, 2000, 395-404.

[5] K. E. Lauter and K. E. Stange, 'The elliptic curve discrete logarithm problem and equivalent hard problems for elliptic divisibility sequences', Proc. 15th Workshop on Selected Areas in Cryptography, Lecture Notes in Comput. Sci., vol. 5381, Springer-Verlag,Berlin, 2009, 309-327.

[6] M. Perret, 'Multiplicative character sums and Kummer coverings', Acta Arith., 59 (1991), 279-290.

[7] A. C. Russell and I. E. Shparlinski, 'Classical and quantum algorithms for function reconstruction via character evaluation', J. Compl., 20 (2004), 404-422.

[8] I. E. Shparlinski, 'Distribution of nonresidues and primitive roots in recurrent sequences', Matem. Zametki, 24 (1978), no. 5, 603-613, (in Russian).

[9] J. H. Silverman, The arithmetic of elliptic curves, 2nd ed., Springer-Verlag, Berlin, 2009.

[10] J. H. Silverman, ' $p$-adic properties of division polynomials and elliptic divisibility sequences', Math. Annalen, 332 (2005), 443-471.

[11] C. Swart, 'Elliptic curves and related sequences', PhD thesis, Royal Holloway and Bedford New College, University of London, 2003.

[12] M. Ward, 'Memoir on elliptic divisibility sequences', Amer. J. Math., 70 (1948), 31-74.

Department of Computing, Macquarie University, North Ryde, SydNEY, NSW 2109, Australia

E-mail address: igor@comp.mq.edu.au

Department of Mathematics, Stanford University, 450 Serra Mall, Building 380, StAnFord, CA 94305, USA

E-mail address: stange@math.stanford.edu 